\title{
From Atomic Guideline-Based Recommendations to Complete Therapeutic Care Plans: A Knowledge- Based Approach Applied to Breast Cancer Management
}

\author{
Hicham KOUZ ${ }^{\mathrm{a}}$, Jacques BOUAUD ${ }^{\mathrm{b}, \mathrm{a}}$, \\ Gilles GUÉZENNEC ${ }^{\mathrm{a}}$ and Brigitte SÉROUSSI ${ }^{\mathrm{a}, \mathrm{c}, 1}$ \\ a Sorbonne Université, Université Sorbonne Paris Nord, INSERM, \\ LIMICS, UMR S 1142, Paris, France \\ b AP-HP, DRCI, Paris, France \\ ${ }^{\mathrm{c}}$ AP-HP, Hôpital Tenon, Paris, France
}

\begin{abstract}
How textual clinical practice guidelines are written may have an impact on how they are formalized and on the kind of recommendations issued by the clinical decision support systems (CDSSs) that implement them. Breast cancer guidelines are mostly centered on the description of the different recommended therapeutic modalities, represented as atomic recommendations, but seldom provide comprehensive plans that drive care delivery. The objective of this work is to implement a knowledge-based approach to develop a care plan builder (CPB) that works on atomic recommendations to build patient-centered care plans as sequences of chronologically ordered therapeutic steps. The CPB uses the atomic recommendations issued by the guideline-based decision support system (GL-DSS) of the DESIREE project. The domain knowledge is represented as the list of all care plans that apply to breast cancer patients. Scenarios are introduced to locate the patient on these theoretical care plans. The CPB has been evaluated on a sample of 99 solved clinical cases leading to an overall performance of $89,8 \%$.
\end{abstract}

Keywords. Decision support systems, Clinical Practice Guidelines, Patient Care Planning, Breast Cancer.

\section{Introduction}

Breast cancer is the most common cancer among women worldwide. In France, the mortality rate of breast cancer is decreasing, which is partly due to the early stage of the disease at diagnosis, and the progress of therapeutic drug protocols. However, although studies have reported that following clinical practice guidelines (CPGs) does improve survival rates of patients [1], the compliance rate of multidisciplinary tumor board (MTB) decisions remains variable. Guideline-based decision support systems (GLDSSs) have been developed to promote MTB implementation of CPGs [2].

\footnotetext{
${ }^{1}$ Corresponding Author, Brigitte Séroussi, DSP, Hôpital Tenon, 4 rue de la Chine, Paris, France; E-mail: brigitte.seroussi@aphp.fr
} 
DESIREE is a European-funded project ${ }^{2}$ that aims at developing a web-based platform for the management of primary breast cancer. It offers different decision support modalities to support the decision at the various stages of patient care, from diagnosis to treatment and follow-up [3]. However, the GL-DSS of DESIREE mostly produces "atomic" recommendations, i.e., recommendations that are focused on one therapeutic modality like surgery or chemotherapy. Such recommendations are regularly redundant, sometimes conflicting, and very rarely organized as comprehensive care plans.

To answer MTB physicians' needs for operational decision support, we have developed a "Care Plan Builder" (CPB) that relies on a knowledge-based approach to build the recommended care plans as a sequence of chronologically ordered therapeutic steps from the atomic recommendations generated by the GL-DSS of DESIREE.

\section{Material and Methods}

\subsection{DESIREE atomic recommendations}

The Breast Cancer Knowledge Model (BCKM) represents the central element for the DSS components of DESIREE. It describes in a common ontology following the EntityAttribute-Value model both the data model and the termino-ontological knowledge used for representing breast cancer concepts and clinical cases. Relying on BCKM concepts, and decision rules that model CPG contents, the GL-DSS produces patient-specific recommendations as atomic recommendations (see Figure 1) at different levels of abstraction (surgery, but also lumpectomy; radiotherapy, but also radiotherapy of the lymph nodes; chemotherapy, but also 3-4 cycles of Epirubicin, etc.). Each recommendation has a level of conformance that may be either positive (SHALL, SHOULD, MAY, and MAYNOT) or negative (SHALLNOT, SHOULDNOT).

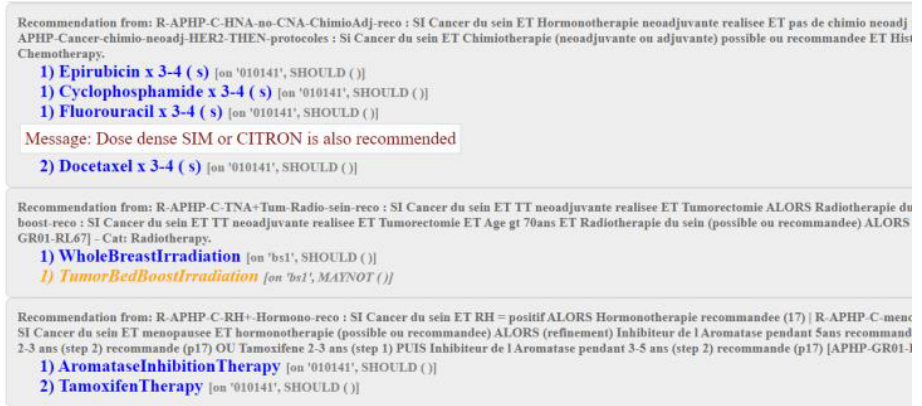

Figure 1. Example of three atomic recommendations issued by the GL-DSS of DESIREE, one of chemotherapy, one of radiotherapy, and one of endocrine therapy.

\subsection{Care plans models for breast cancer management}

Cancer care plans are organized around a number of treatment methods such as surgery (SUR), chemotherapy (CHEM), targeted therapies, endocrine therapy (HO), and radiotherapy $(\mathrm{RAD})$. The diagram displayed in Figure 2 illustrates all the possible

\footnotetext{
${ }^{2}$ The DESIREE project has received funding from the European Union's Horizon 2020 research and innovation programme under grant agreement No 690238.
} 
trajectories for the management of non-metastatic breast cancer patients, from the moment the diagnosis is made, and passing through the different scenarios $(\mathrm{A}, \mathrm{B}, \mathrm{C}$, and D). Each path can have a single step method or an ordered combination of methods defined by a branch.

\section{A : initial decision \\ B : decision after neoadjuvant treatment (NAT) \\ C: decision after surgery and neoadjuvant treatment (SUR \& NAT) \\ D : decision after surgery without neoadjuvant treatment (SUR without NAT )}

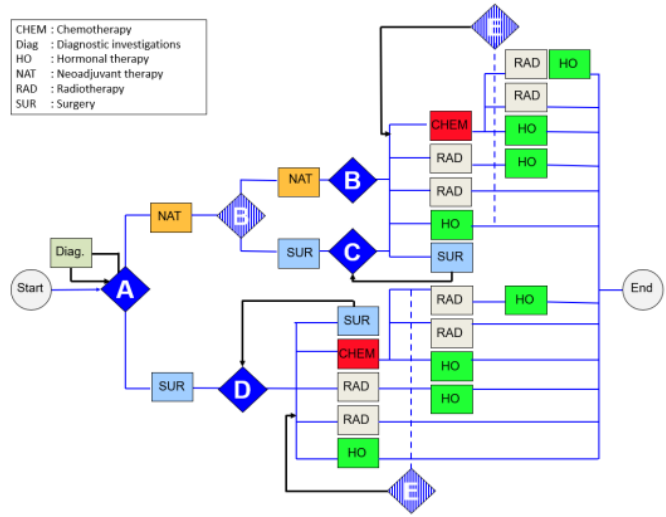

Figure 2. Diagram of all medically relevant care plans for breast cancer patients.

\subsection{Care plan representation}

To represent the structure of the care plan model, we borrowed from the conceptual frameworks of existing data models, e.g., FHIR [4] and Open EHR [5]. From FHIR, we used the "CarePlan" resource to represent the general information of a patient (identifier, date, etc.), and the "PlanDefinition" resource to represent a group of actions defined as a step in our care plan. We also used the "Task plan" model of "Open EHR task Planning" for the definition of each action named "activity".

\subsection{Care plan building processes}

We proceeded in six stages to build the care plans from atomic recommendations:

1. Analysis of recommendations generated by the GL-DSS, identification of R+, defined as the set of recommendations with a positive conformance level and $\mathrm{R}$-, defined as the set of recommendations with a negative conformance level;

2. Elimination of conflicting recommendations that exist when there is a positive conformance level (e.g., Tumorectomy SHOULD) and a negative conformance level (BreastSurgery SHOULDNOT) taking into account the subsumption relationship. The process consists in browsing all the recommendations of $\mathrm{R}+$ in order to check if there is a comparable recommendation in $\mathrm{R}$ - and to proceed with the elimination of both of them;

3. Elimination of recommendations that have a negative conformance level which are not useful for the construction of care plans since only those with a positive conformance level have to be actually performed;

4. For each recommendation, identification of the corresponding therapeutic category (chemotherapy, endocrine therapy, radiotherapy, surgery) to reach the level of abstraction of the care plan models (see 2.2); 
5. Taking into account the therapeutic categories previously identified and the scenario of the patient management, identification of the model of care plan from the set of all possible care plans (see 2.2);

6. Generation of instantiated care plans based on the remaining recommendations and the model identified in the previous stage.

The CPB has been assessed on a sample of solved clinical cases for which we had (i) the set of atomic recommendations issued by the GL-DSS, (ii) the MTB decision expressed as a care plan, and (iii) the compliance status of the MTB decision with CPGs as previously established by clinicians (gold standard). The CPB performance was defined by the frequency with which MTB decisions acknowledged as compliant with CPGs were retrieved in the care plans generated from the atomic recommendations of the GL-DSS.

\section{Results}

Figure 3 illustrates how care plans are built from atomic recommendations. We use the case of a patient in "scenario C" that generated seven recommendations among three therapeutic categories (chemotherapy with three instances, radiotherapy with one instance, and endocrine therapy with three instances), and the care plans generated by the CPB (an excerpt with four out of the nine care plans generated is displayed).

We used a sample of 99 clinical cases with CPG-compliant MTB decisions that were solved using DESIREE to produce atomic recommendations processed by the CPB. For 89 clinical cases, the MTB decision was found in the list of CPB-generated care plans, which corresponds to an overall performance of $89.8 \%$.
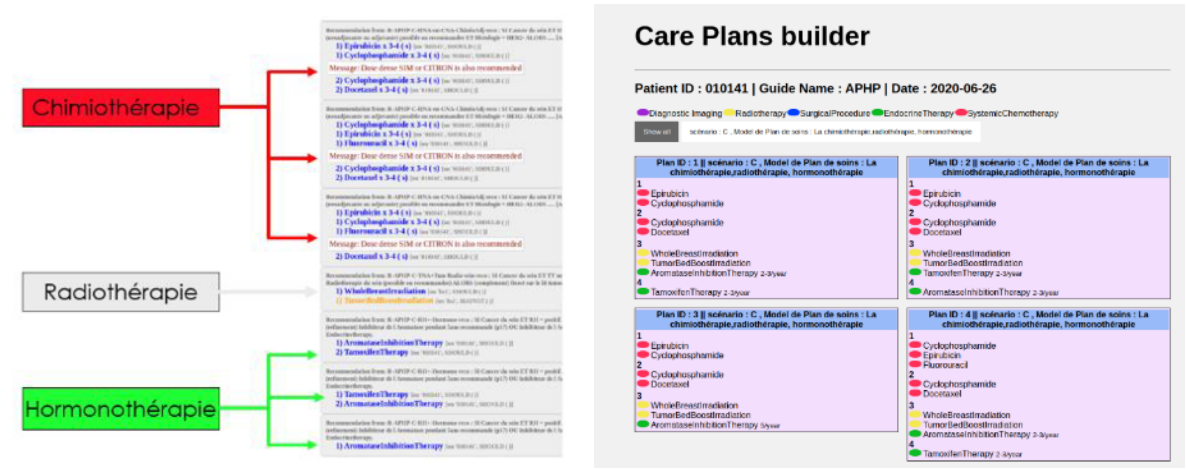

Figure 3. Care plans generated from DESIREE atomic recommendations in scenario C.

\section{Discussion and Conclusion}

Not all GBPs recommend comprehensive care plans that can be directly encoded for decision support [6]. We have developed a care plan builder allowing the consistent processing of atomic recommendations issued by the GL-DSS of DESIREE to generate the corresponding recommended complete care plans. The CPB gives satisfactory results with a performance of $89.8 \%$. For 10 clinical cases, the MTB decision was not found 
among the care plans generated. In seven cases, DESIREE outputs were at the origin of the issue (a MTB decision was badly entered; some chemotherapies were missing in the recommendations $(n=3)$; target therapies wrongly included in the BCKM as sort of chemotherapies $(n=3))$. Only three badly processed cases were imputable to the CPB due to a mismanagement of the subsumption relationship.

Despite being focused on one pathology (breast cancer) and on one guideline, building care plans from atomic recommendations is part of the general scientific research topic on guideline reconciliation, e.g., for the management of multimorbidity. In these situations, CDSSs generate several recommendations that might conflict or may be combined, and for which varied approaches have been proposed (see [7]). In our case, it is as if we had several guidelines, one per therapeutic modality, and the additional knowledge used to build care plans (e.g., no chemotherapy after radiotherapy) can be considered as constraints to be satisfied in building the care plan.

This work has some limitations. We based the CPB development on the assumption of atomic recommendations, allowing to use the FirstStepCategory. However, in some cases, so-called atomic recommendations were in fact semi-care plans. The resolution of conflicts (removing pairs of similar recommendations that had a positive conformance level for one of them and a negative conformance level for the other one) is a pragmatic and empirical approach but it means that the negative conformance level is favored which should be fine-tuned by considering additional domain knowledge (to select the recommendations to be removed instead of removing them both). Finally, the definition of the performance measure has imperfections. Indeed, we considered the frequency with which MTB decisions acknowledged as compliant with CPGs were retrieved in the care plans generated from the atomic recommendations of the GL-DSS. Thus, we do not have any evaluation of the CPB when MTB decisions were not compliant with CPGs and we didn't evaluate the generated care plans that were different from the compliant MTB decision. Further work is needed to improve the CPB (to take into account non-atomic recommendations) and the performance indicator.

\section{References}

[1] Kreienberg R, Wöckel A, Wischnewsky M. Highly significant improvement in guideline adherence, relapse-free and overall survival in breast cancer patients when treated at certified breast cancer centres: An evaluation of 8323 patients. Breast. 2018;40:54-59.

[2] Eccher C, Seyfang A, Ferro A. Implementation and evaluation of an Asbru-based decision support system for adjuvant treatment in breast cancer. Comput Methods Programs Biomed. 2014;117(2):308-321.

[3] Seroussi B, Lamy JB, Muro N, et al. Implementing Guideline-Based, Experience-Based, and Case-Based Approaches to Enrich Decision Support for the Management of Breast Cancer Patients in the DESIREE Project. Stud Health Technol Inform. 2018;255:190-194.

[4] HL7. CarePlan - FHIR v4.0.1. [available at https://www.h17.org/fhir/careplan.html]

[5] Anani N, Chen R, Prazeres Moreira T, Koch S. OpenEHR-based representation of guideline compliance data through the example of stroke clinical practice guidelines. Stud Health Technol Inform. 2012; 180:487-491.

[6] Laleci Erturkmen GB, Yuksel M, Sarigul B, et al. A Collaborative Platform for Management of Chronic Diseases via Guideline-Driven Individualized Care Plans. Comput Struct Biotechnol J. 2019;17:869-885.

[7] Bilici E, Despotou G, Arvanitis TN. The use of computer-interpretable clinical guidelines to manage care complexities of patients with multimorbid conditions: A review. Digit Health. 2018;4:2055207618804927. 